\title{
ECOULEMENT EN MILIEU POREUX FUITES SOUS LES BARRAGES
}

\section{G. SAUVAGE DE S' MARC}

Ingénieur des Arts ef Manufactures

Nous n'avons pas le dessein, dans cet article, d'examiner le problème des fuites sous les barrages dans toute sa généralité. Nous nous borne'rons à déterminer l'écoulement souterrain dans quelques cas schématiques que la théorie nous a permis de résoudre et qui présentent, nous semble-t-il, un grand jntérêt pour les Ingénieurs chargés d'établir des projets de barrages fondés sur des terrains perméables.

C'est donc à un travail théorique que nous nours sommes livrés; mais voulant réduire le plus possible les calculs relatifs aux fonctions analytiques, nous avons ell recours à des mélhodes géométriques mises en honneur et largement exploitées par MM. BARILLon et DANEL. Evidemment, pour comprendre parfaitement les diverses transformations géométriques utilisées, dans notre exposé, le lecteur devra se reporter à différents ouvrages de base ( ${ }^{r}$ ) ; cet écueil ne nous a pas échappé, aussi avons-nous tenté, en guise de prélude, de présenter ces transformations sous une forme très intuitive, généralement condamnée par les mathématiciens, $m$ a i $s$ appréciée par l'ingénieur, surtout si son esprit se tourne volontiers vers la recherche géométrique.

Ces remarques préliminaires paraitront un peu abstraites; cependant, nous avons cru devoir les placer en tête de cette rapide étude pour bien préciser notre méthode et notre but, en espérant ne pas lasser le lecteur avant que d'avoir commencé.

\section{RAPPEL DES PRINCIPALES PROPRIETES}

\section{DES ECOULEMENTS EN MLLIEU POREUX}

Les écoulements en milieu poreux se caractérisent par un petit nombre de Reynolos, dû à l'extrême faiblesse de la vitesse. PRANitr les qualifie «d'écoulements rampants ». La vitesse

(1) Cours de M. Barruon à l'Ecole du Génie Maritime. Cours des Elèves Ingénieurs hydrauliciens de Grenoble. apparente en point M, (débit divisé par la section du sol), est donnée par la loi de Darcy :

$$
\mathrm{V}=k \frac{d \mathrm{H}}{d s}
$$

où $k$ désigne une constante appelée perméabilité et ayant les dimensions d'une vitesse ; $\mathbf{H}$ représente la charge au point $\mathrm{M}$, et $\ll d \mathrm{H} \gg$ la perte de charge sur le parcours $《 d s \gg$ le long de la ligne de courant passant par M. La perte de charge est donc proportionnelle à la vitesse.

On voit que $V$ dérive du potentiel $\Phi=k \mathrm{H}$; le réseau d'écoulement est donc isotherme, c'està-dire représentable par une fonction analytique ; les lignes équipotentielles sont des lignes d'égale charge (ou d'égale hauteur piézométrique).

Ces généralités rapidement rappelées, s’appliquent dans un milieu infini ; la réalité fait apparaître certaines limites; sur ces limites doivent être respectées certaines conditions; par exemple, une paroi ou un fond imperméable se confond avec une ligne de courant; ou bien encore, les infiltrations se font généralement par une ligne de séparation entre l'eau et le terrain; cette ligne de séparation constitue une équipotentielle et toutes les lignes de courant partent normalement à cette ligne.

Cet apercu nous semble suffisant pour aborder maintenant les problèmes en vue, que nous ferons précéder par la présentation de quelques transformations conformes.

\section{NOTIONS SUR QUELQUES \\ TRANSFORMATIONS PARTICULIERES}

$\left.1^{\circ}\right)$ Transformation dite des charnieres.

Analytiquement, elle s'écrit: $Z=\arccos z$. Dans le plan des $z$, la fonction Arc cos $z$ représente le réseau des ellipses et hyperboles homofocales (foyers communs $M$ et $M^{\prime}$ ). Dans le plan $Z$, la fonction de $Z$ représente un damier. A chaque hyperbole correspond donc une droite verticale du damier ; le nombre des hyperboles étant limité, le nombre des droites 


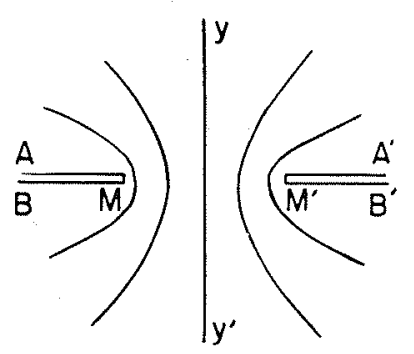

plan 2

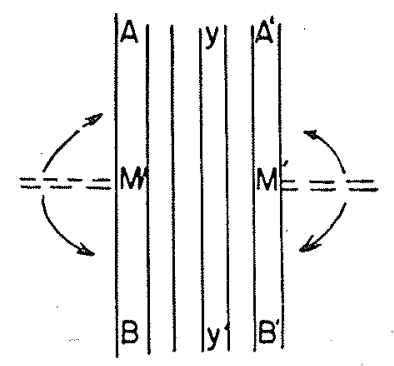

plan $Z$
Fig. 1

verticales du damier est également limité ; la transformation se fera donc entre la totalité du plan des $z$ et une bande du plan des $Z$.

Le segment MM' ne change pas de nature dans la transformation ; l'hyperbole droite correspondant à $y y^{\prime}$ reste la droite yy'. L'hyperbole $A M B$ et A'M'B' devient deux droites verticales limitant la bande; les demi-droites AM et BM d'une part, A'M' et B'M' d'autre part, pivotent de $\frac{\pi}{2}$ dans des sens différents autour des points M et M' qui jouent ainsi le rôle de charnières d'où le nom que nous avons choisi pour désigner cette transformation. Désormais, pour guider notre intuition, nous verrons dans cette transformation :

- l'invariance du segment $\mathrm{M}$ M' et de $y y^{\prime}$ d'une part,

- le pivotement autour des charnières M et M' d'autre part (2).

(2) Nous ne voulons pas dire, par la, que les points du segment MM' ne bougent pas dans la transformation, mais simplement qu'ils se retrouvent avec la même disposition relative sur le segment MM', apres transformation.

De mème les points de la demi-droite $A M$ se retrouvent dans le même ordre sur la demi-droite transformée. Notons d'ailleurs que tous ces points (ceux de MM' comme ceux de $A M$ et de $A^{\prime} M^{\prime}$ ) sont situés à égale distance les uns des autres sur le plan des $Z$.
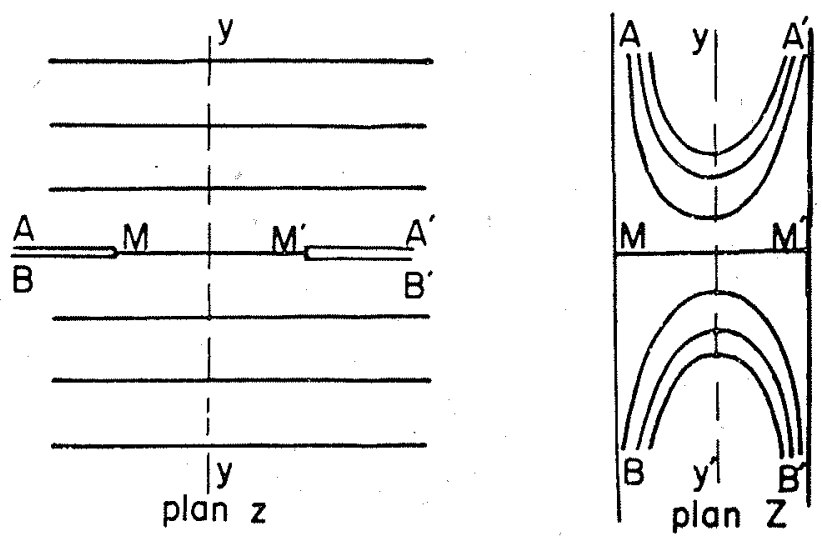

Fig. 2

\section{$\left.2^{\circ}\right)$ Réseau réciproque d'un réseau}

La transformation précédente peut être définie autrement que par la correspondance entre uo damier et les hyperboles homofocales. Prenons, par exemple, la fonction inverse $z=\operatorname{Cos} Z$. Au réseau $\cos Z$ dans le plan des $Z$ correspond un damier dans le plan des $z$. Le réseau $\cos Z$ est dit réciproque des hyperboles homofocales. D'une manière plus générale, la fonction inverse représente le réseau réciproque du réseau initial correspondant à la fonction directe. Géométriquement, soit un réseau $R$ dans un plan $P$ et un damier $D$ dans un plan $\Pi$. Placons dans le plan $\mathrm{P}$ un damier $d$ et transformons par $(\mathrm{R}-\mathrm{D})$; nous obtenons un
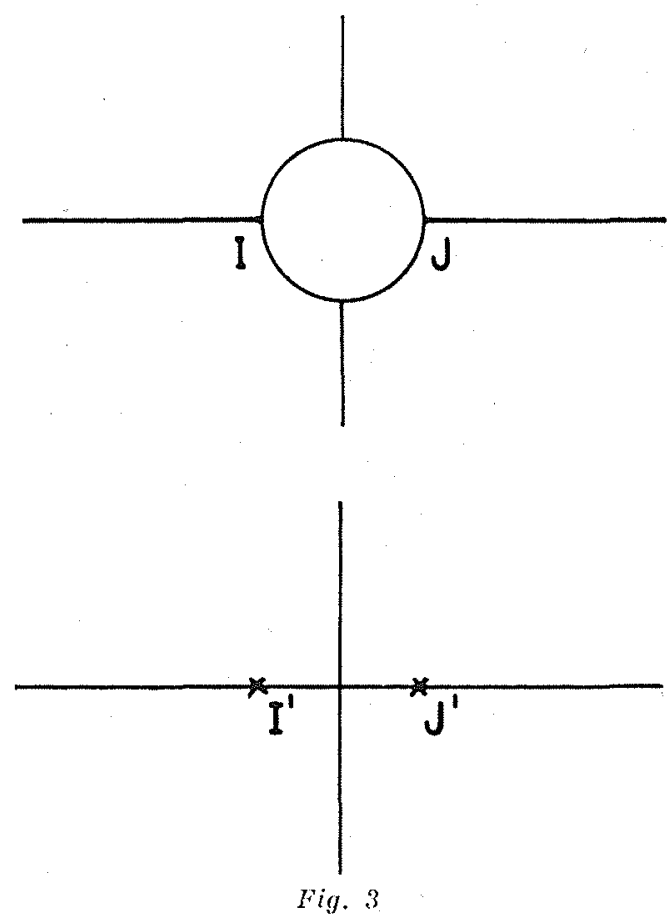

réseau $r$ dans $\Pi$ I $r$ est le réciproque do R.

Cette notion de réciproque n'est qu'à peine définie ici; nous aurions voulu la développer davantage, car son importance s'affirme chaque jour plus clairement à nos yeux, mais nous sortirions du cadre que nous nous sommes fixé.

$\left.3^{\prime \prime}\right)$ Transformation de Joukowsky.

Elle est trop connue.pour que nous en redonnions une définition analytique. Nous plaçant toujours de notre point géométrique, nous retiendrons certaines particularités qui, au cours d'une étude, pourront nours faire songer à appliquer cette transformation. Une Joukowsky ayant comme points principaux $I$ et $J$, transforme tout l'extérieur du cercle $\mathrm{C}$ de diamètre I J en un plan complet; le cercle ${ }_{i} \mathrm{C}$ s'aplatit sui- 
vant I' J'; ce passage du cercle $C$ au segment I' J' nous servira. Par exemple, considérons l'écoulement autour d'un cercle placé dans un écoulement parallèle. Une Joukowsky de points principaux I $J$ transforme ce réseau en un damier. Sans doute, le lecteur pensera que nous jouons à l'illusionniste, et que nous exécutons devant lui un tour de passe-passe. Qu'il s'entraîne à cette gymnastique, et toutes ces transformations deviendront, pour lui, un jeu très agréable.

Ces notions tres sommaires vont nous permettre d'aborder maintenant les cas pratiques que nous avons annoncés au début.
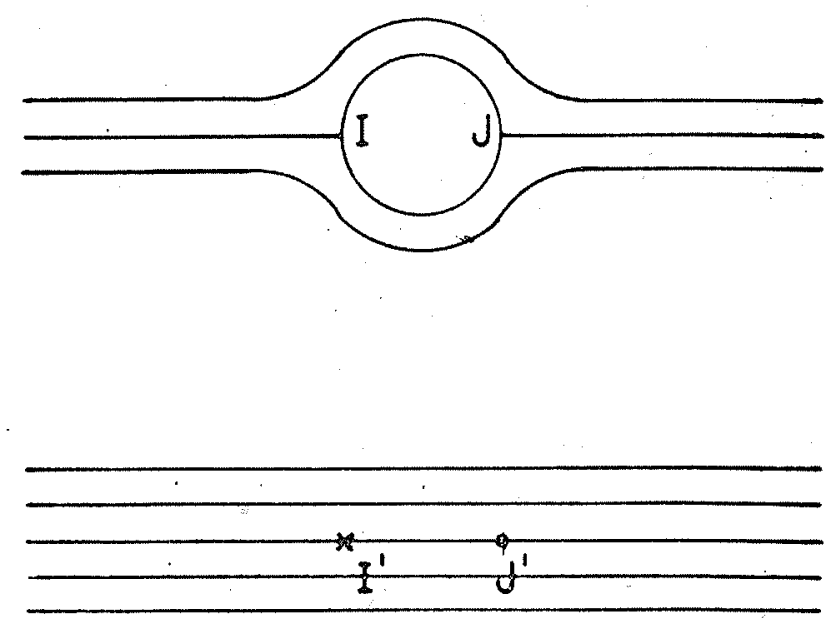

Fig. 4

\section{FUITES SOUS ILES BARRAGES}

Dans la nature, l'assise d'un barrage peut affecter des formes différentes ; pour la simplicité de notre exposé, nous distinguerons deux catégories : une première où l'assise du barrage se réduit à un simple radier horizontal $B$ C étanche; une deuxième où le radier horizontal est complété à l'amont ou à l'aval par des écrans d'étanchéité.

Dès lors, nous étudierons successivement le cas du radier horizontal seul, le cas de l'étanchéité seul, et enfin le cas le plus général, comportant radier et écran d'étanchéité.

I - CAS du RADIER horizonTAL SEUl

La figure indique le schéma que nous avons en vue ; la base $B C$ est imperméable.

Nous supposerons le terrain homogène de perméabilité $k$. Le réseau d'écoulement pourra être influencé par la présence d'un substratum imperméable.

, Lorsque nous considérons un milieu infini, c'est-à-dire lor'sque cette couche étanche n'exisie pas, le probleme est bien connu; nous avons
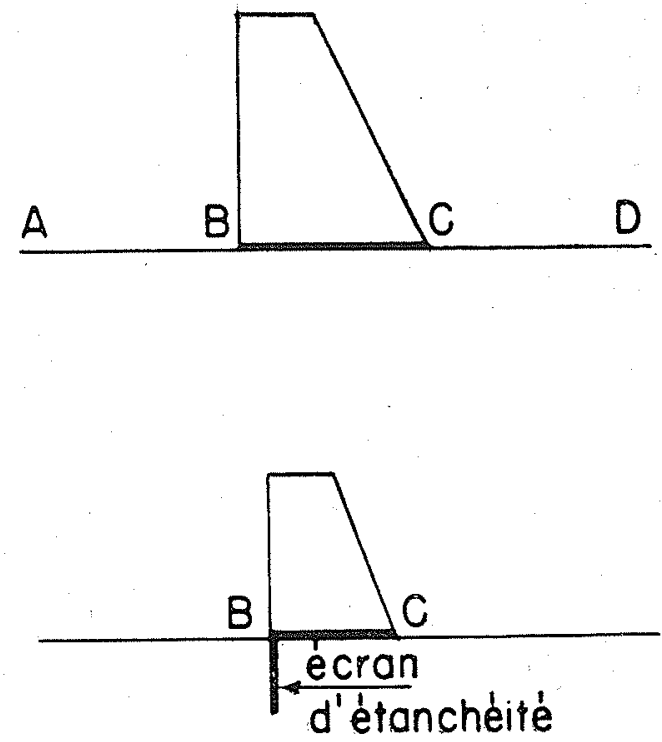

Fig. 5

alors afraire au réseau isotherme des éllipses et des hyperboles homofocales (les foyers communs sont B et C). Nous nous attacherons donc à trouver la solution en milieu fini, c'està-dire en présence d'une étanchéité horizontale.

Lorsque le fond imperméable est à distance finie, les lignes de courant s'aplatissent de sorte que nous avons intuitivement une idée du schéma d'écoulement; deux de ces lignes de colrant apparaissent avec évidence : la ligne $B C$ et la ligne EF.

Complétons le par son symétrique par rapport à la ligne AD.

Le problème revient donc à passer de ce schéma que nous ne savons pas encore construire, à un réseau connu soit par son expression analytique, soit par son tracé géométrique.

Effectuons alors une transformation des charnières de base, $G G^{\prime}$, les points $G G^{\prime}$ étant les extrémités du deuxième axe de symétrie de la figure. Notre schéma se transforme en la figure "n" 9. A nouveau, déformons encore ce

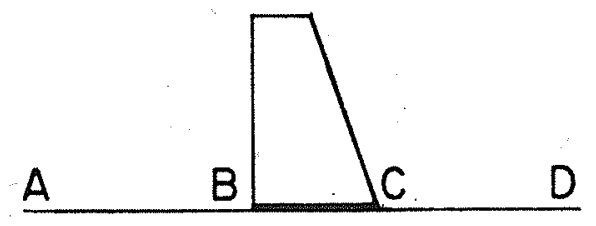



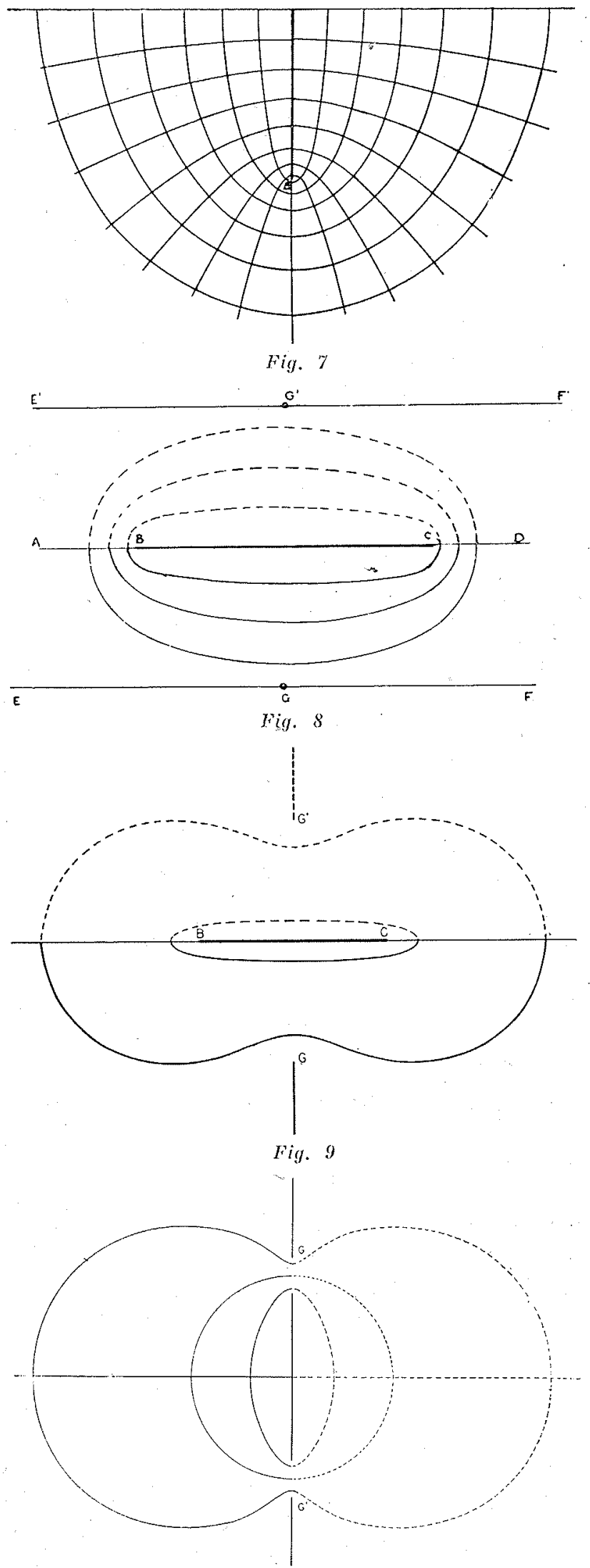

Fig. 10 réseau à l'aide de l'inverse d'une Joukowsky de maniere à faire disparâtre le segment rectiligne B C. Prenons comme points principaux de la transformation, les points $B$ et $C$. Le segment B C se dilate suivant un cercle el nous obtenons le réseau indiqué sur la figure. Ce réseau représente la fonction inverse d'une fonction elliptique $s n z$, ou avec notre convention de langage, ce réseau est le réciproque du sin $z$.

Partant donc de ce réciproque (3) et remontant la série des transformations précédentes, nous parvenons au réseau cherché.

Le tracé de ce réseau nous permel d'évaluer les fuites sous le barrage et d'étudier la stabilité du barrage grace à la courbe des pressions le long de $B$ C.

a) Fuite. - Chaque tube de courant laisse passer un débit égal à

$$
\mathrm{V} d s=\mathrm{K} \frac{d h}{d s} d s=\mathrm{KdH}
$$

Si « $n$ ' est le nombre de tubes de courant, c'est-à-dire le nombre de carreaux le long de $A B$, le débit de fuite sera :

$$
\mathrm{Q}=n^{\prime} k^{\prime} d \mathrm{H}
$$

Comme les lignes équipotentielles sont des lignes d'égale charge, la différence de charge entre deux équipotentielles sera $d \mathrm{H}=\frac{\mathrm{H}}{n}$ où $n$ représente le nombre de carreaux le long de $B$ C. Dès lors, l'expression du débit de fuite devient :

$$
\mathrm{Q}=k \mathrm{H} \frac{n !}{n}
$$

b) Courbe de pressions. - Le réseau une fois tracé, il devient aisé de déterminer les pressions le long de $B$ C, à partir de la dimension des carreaux adjacents à B. C.

Nous laissons au lecteur, le soin de voir par lui-mème l'allure de ces courbes : signalons seulement que dans le cas d'un milieu infini, cette courbe des pressions est une sinusoïde.

\section{II - CaLS DE L'ÉCRAN d'ÉTANChétté SEUL}

Comme pour le cas précédent, le réseau en milieu infini ne pose pas de difficultés particulières; c'est encore le réseau des ellipses el hyperboles homofocales (les foyers sont le point $C$ et son symétrique C' par rapport à A E). Mais là encore, ce cas n'intéresse que fort peu l'ingénieur: car il ne se présente que rarement dan's la pratique.

(3) Co réciproque de sn $z$ peut se construte geometriquement (voir cours de l'E.I.H.). 
Forscheimer a étudié le problème en milieu fini ; il en a donné une solution approchée (t) confrontée avec l'expérience par $R$. Howl MANN (s). Nous présenterons, ici, deux solutions exactes, que notre méthode géométrique nous a suggérées.

Première solution. - Partons du réseau représenté par la fonction inverse de isn $z$; ce réseau que nous avons rencontrè tout à l'heure, admet l'axe $y y^{\prime}$ comme axe de symétrie ; effectuons une tranformation des charnières $G G$ ' et nous obtenons le réseau cherché. Il est possible, à partir de lá, de connaître l'expression analytique de ce champ; nous n'entreprendrons pas ce travail ici.

Nous préférons donner une autre solution qui nous permettra des conclusions intéressantes relatives au débit de fuite.

Deuxième solution. - Partons du champ d'un vortex placé au milieu d'un rectangle, champ que nou's désignerons par $V$. Si nous considérons le réciproque de ce champ (c'est-à-dire la fonction inverse) nous tombons sur le réseau cherché. Il est alors possible de trouver assez rapidement l'expression analytique du vortex dans le rectangle; elle serait de la forme (6) :

$$
f(z)=2 \operatorname{Arcos}\left[d n\left(\frac{z}{\sqrt{2}}\right)\right]
$$

Ce détail analytique pourra surprendre le lecteur, mais nous voulons simplement montrer que les fonctions elliptiques interviennent tout naturellement dans notre calcul des débits de fuite.

(4) Compte rendu de l'Académie Impériale des Sciences a Vienne. Section Ira, vol. 126 - 1917.

(5) «Die Wasserwirtschaft » de 1934 - Cahier 18-19 et $20-21$.

(6) dn représente unc notation employée par M. BaRr.. roN pour designer une fonction elliptique.

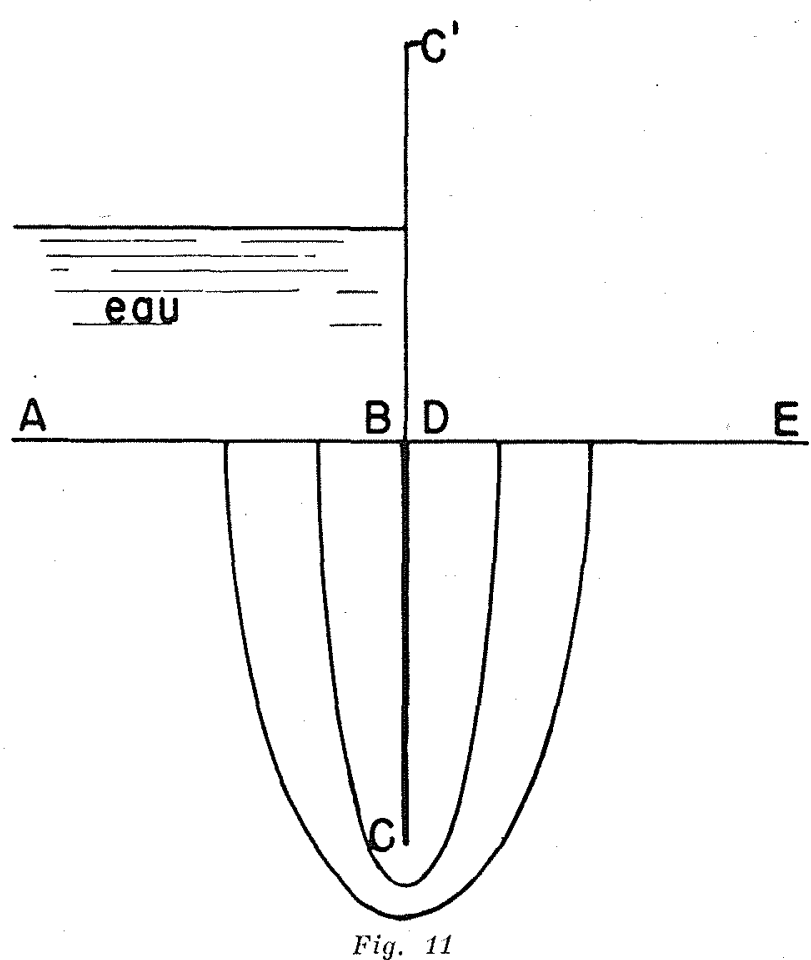

Regardons de plus près la correspondance entre le champ $\mathrm{V}$ et son réciproque, correspondance indiquée par l'emploi de lettres homologues sur la figure ci-contre.

Nous voyons que le réseau réciproque présente une certaine périodicité ; nous nous limiterons à la portion intéressante $A^{\prime} B^{\prime} D^{\prime} A^{\prime}$. La droite A' $B$ ' indique la ligne de séparation entre le terrain poreux et l'eau; de même A' D', celle entre le milieu perméable et le sol imperméable. La palplanche est représentée par B' C' . Evidemment, pour avoir notre schéma d'écoulement sous la palplanche, nous prolongerons, par symétrie, autour de B'D'. Or, d'après ce que nous avons vu précédemment, Ic débil de fuite est donné par l'expression :

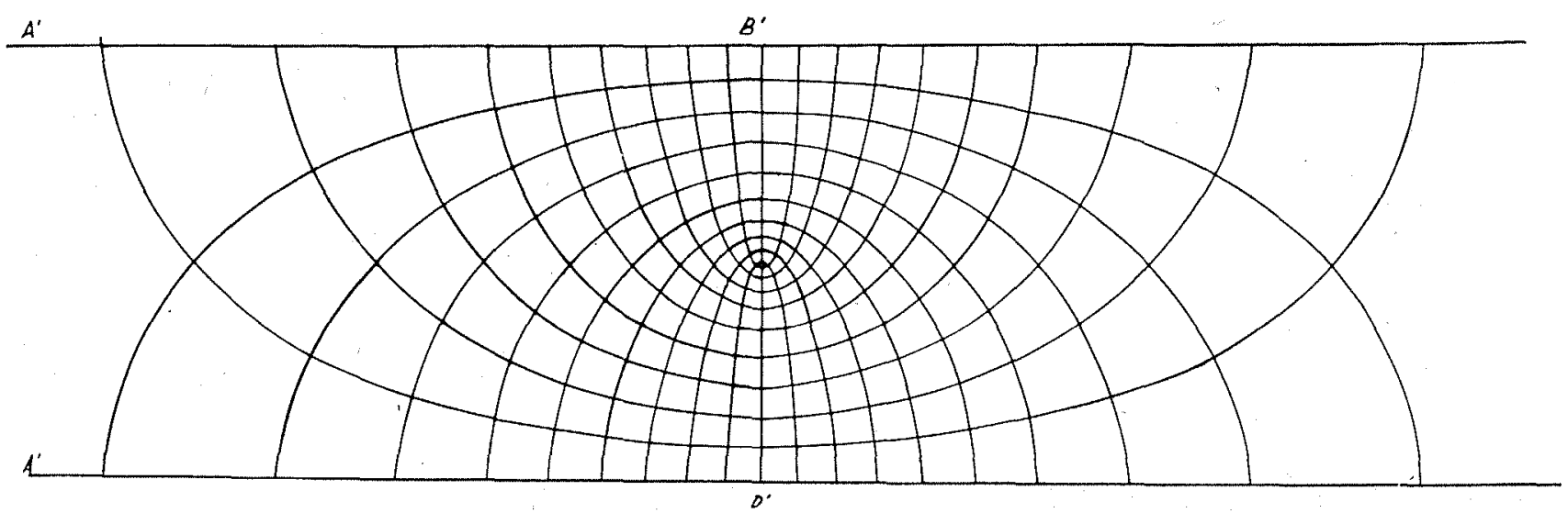

Fig. 12 


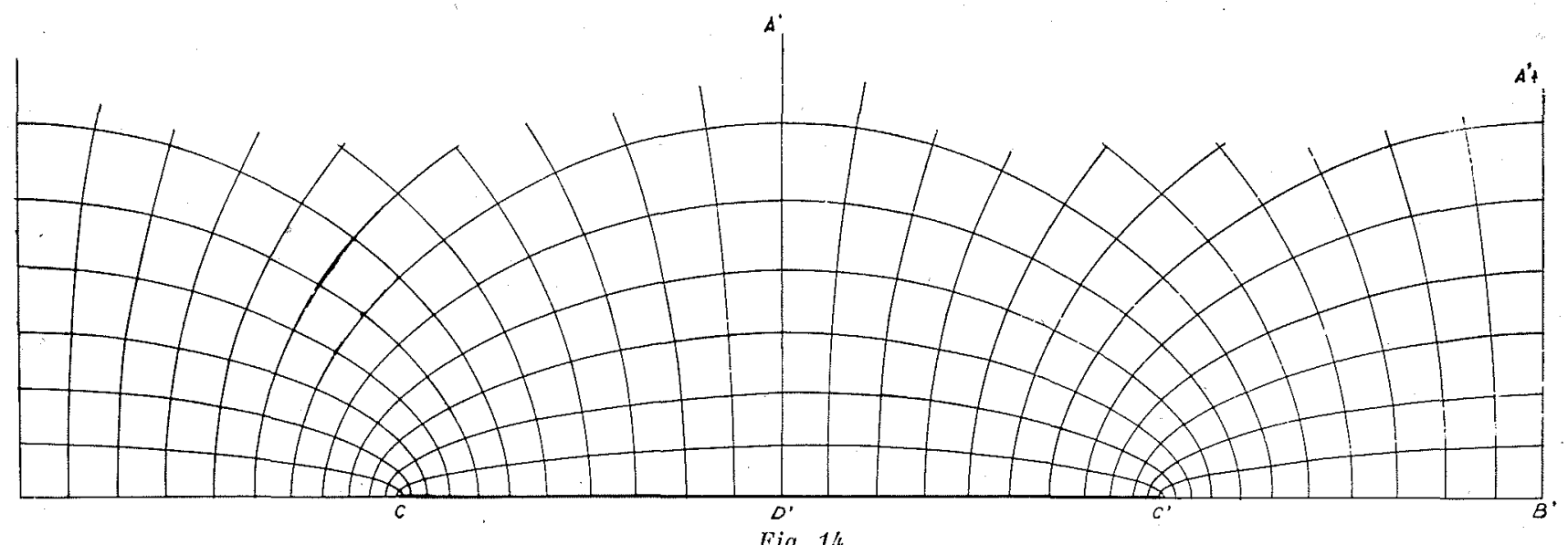

Fig. 14

$$
q=k \mathrm{H} \frac{n^{\prime}}{n}
$$

où $\mathrm{H}$ est la hanteur d'eau au-dessus de A'B', $k$ la perméabilité du terrain,

n' nombre de carreaux le long de $A^{\prime} B^{\prime}$,

$n$ nombre de carreaux le long le B'C' (de part et d'autre de $\mathrm{B}^{\prime} \mathrm{C}^{\prime}$ ).

Dans cette expression, le facteur $\frac{n}{n}$ dépend de la profondeur a laquelle descend la palplanche; nous essayerons done de voir la variation de $\frac{n}{n}$ en fonction du rapport $\frac{l}{\mathrm{~L}}, \mathrm{~L}$ étant la profondeur du sol imperméable et.l celle des palplanches.

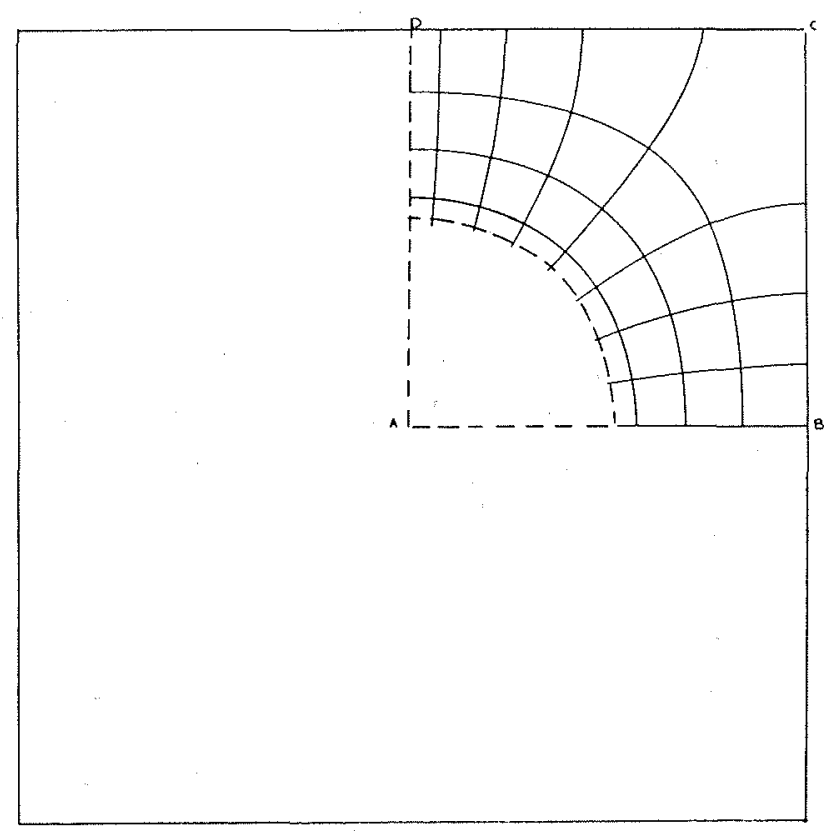

Fig. 13
La nature même des réseaux réciproques nous permet d'établir que le rapport $\frac{l}{\mathrm{~L}}$ est égal au rapport $\alpha$ des nombres de carreaux sur les contours correspondants du champ $V$. L'expres-

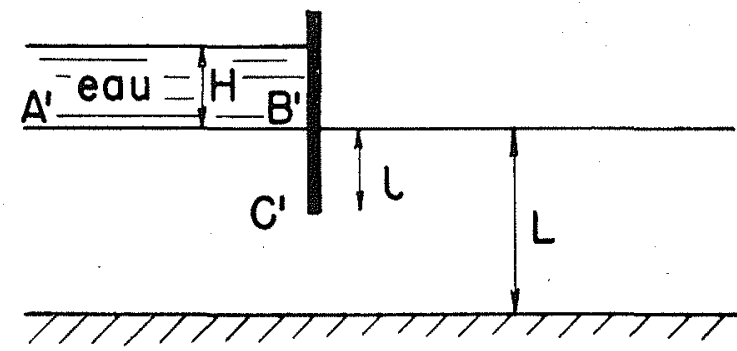

Fig. 15

sion analytique du champ $\mathrm{V}$ donne la possibilité de relier au rapport $\frac{K}{K}, K$ et $K$, étant les expressions courantes dans la théorie des fonctions élliptiques.

$$
\text { (7) } \quad \begin{aligned}
\mathrm{K} & =\int_{0}^{1} \frac{d x}{\sqrt{\left(1-x^{2}\right)\left(1-k^{2} x^{2}\right)}} \\
\mathrm{K}^{\prime} & =\int_{0}^{1} \frac{\operatorname{avec} k^{2}+k^{12}}{\frac{d x_{1}}{\sqrt{\left(1-x_{1}^{2}\right)\left(1-k^{2} x_{1^{2}}\right)}}}
\end{aligned}
$$

Or, $\mathrm{K}$ et $\mathrm{K}$ ' sont donnés par des tables ; nos calculs sont donc simplifiés.

Finalement, la variation du débit avec $\frac{l}{\mathrm{~L}}$ est donnée par la courbe fig. 16 .

L'observation de cette courbe montre l'influen-

(7) Notation de M. Barilron dans son cour's de l'Ecole d'Application du Génie Maritime. $\mathrm{Ne}$ pas confondre $k$ et ' $k$ ' de ces formules avec le $k$ coefficient de permésbilité. 


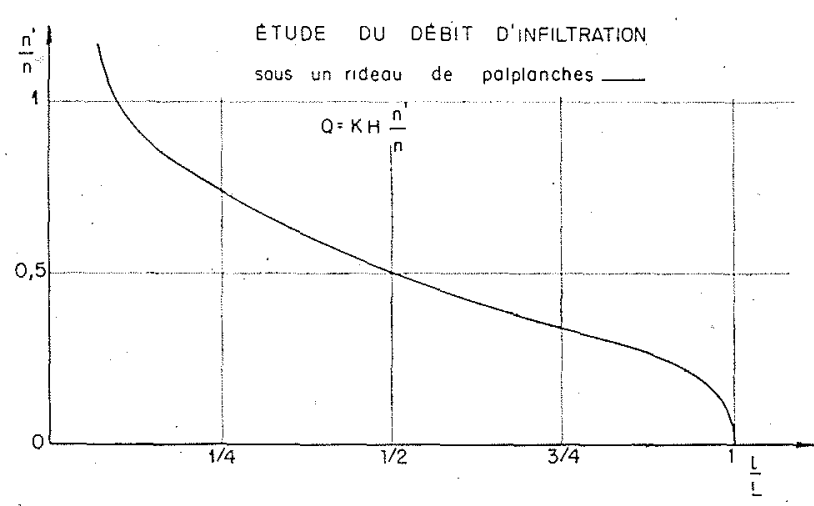

Fig. 16

ce intéressante apportée sur le débit de fuite par un rideau de palplanches, mème peu profond.

Ces premiers résultats peuvent se généraliser, en supposant un décalage entre les terrains en amont et en aval des palplanches.

GENERALISATION. - Nous avons cherché à ramener ce cas à celui que nous venons d'étudier. Cette recherche nous a entraînés dans des études de transformation que nous ne voulons pas développer ici ; nous nous bornerons à en donner les résultats qui seuls présentent un intérêt pour la suite de notre exposé.

Considérons les deux champs figurés sur les; figures ci-contre :

Le premier peut être appelé le réseau des hyperboles périodiques; il convient bien de noter que ces lignes de courant ne sont pas de's hyperboles, mais ce réseau se déduit des hyperboles homofocales par une transformation des charnières; quant au second, nous le désignerons du nom de réseau plaque. Il est également déduit des hyperboles homofocales par une transformation des charnières mais avec d'autres points de base. Ils sont done tous deux aisés à construire. Superposons alors les plans de ces deux réseaux de la manière indiquée par notre schéma.

Sorent $M$ le point du reseau plaque et $\mathrm{K}$ le point correspondant (c'est-à-dire de même coordonnée isothermique $\Phi$ et $\Psi$ ) du réseau des hyperboles périodiques. Joignons $\mathrm{K} \mathrm{M}$ et prolongeons ce segment d'une longueur égale à lui-mème, toujours dans le sens $\mathrm{K}$ M ; le point $K$ ' ainsi obtenu décrit le réseau isotherme $R$.

Ainsi s'établit une correspondance entre B C D du réseau $R$ et B'C'D' du réseau plaque, c'est-à-dire du réseau étudié précédemment. Le rapport $\frac{n}{n}$ considéré dans le réseau plaque ne change pas dans la correspondance ; il sufft de déterminer le rapport $\frac{l}{\mathrm{~L}}$, du réseau plaque permettant de construire le réseau $R$ satisfaisant aux grandeurs $L_{1}, L_{2}$, et $l$. Cette détermination se fait par un calcul analytique qu'il est inutile de présenter ici. Les résultats se trouvent résumés dans l'abaque de la figure.

Le tracé effectif du réseau se fait par la méthode résolvant le cas général étudié plus loin. La figure 23 donne le réseau obtenu dans un cas particulier.

III - Cas génÉral : COMbINaison dU RADIER HORIZONTAL ET D'UN ÉCRAN D'ÉTANCHÉTTÉ

Le réseau des ellipses périodiques du $1^{\text {er }}$ paragraphe ainsi que le réseau $R$ de la généralisation précédente, vont nous permettre de tracer les réseaux d'écoulement que nous avons en vue.

Le réseau $R$ comprend un certain nombre de lignes de courant; faisons correspondre à ces lignes, des droites paralleles équidistantes; aux équipotentielles correspondront également des droites parallèles équidistantes, normales
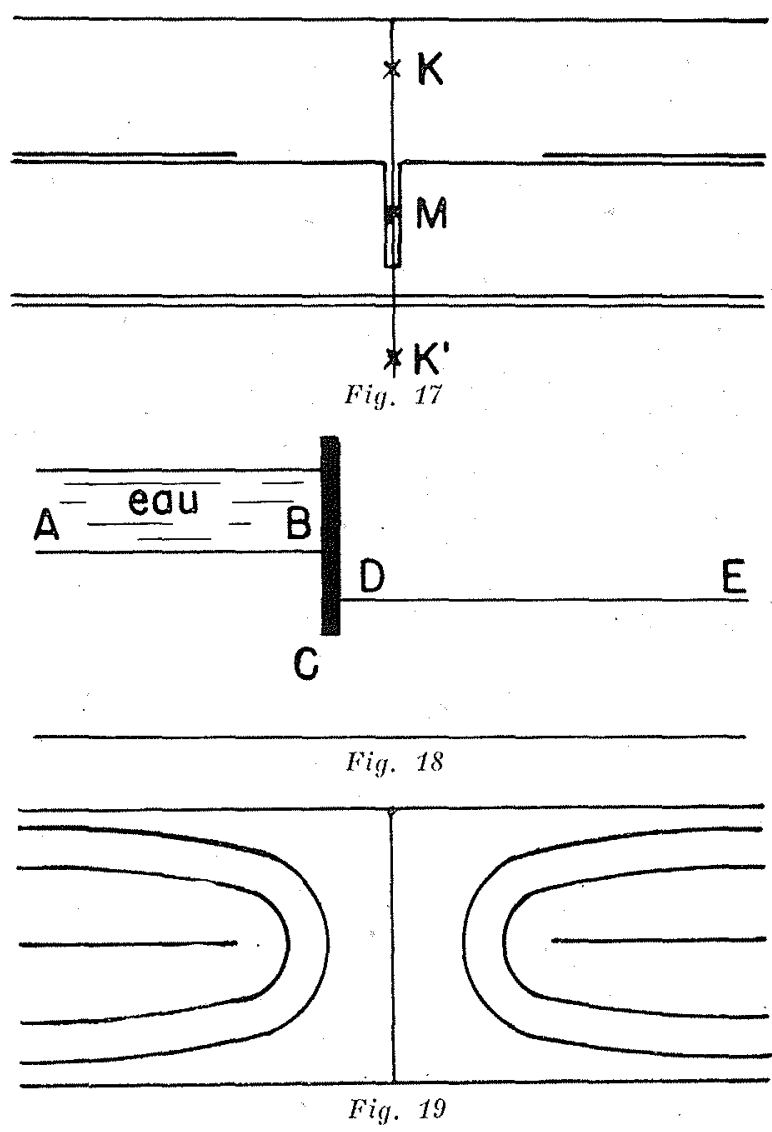

$B^{\prime} D^{\prime}$

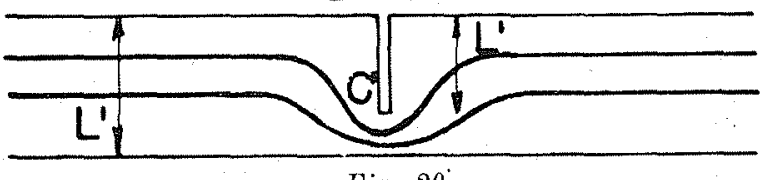

Fig. $20^{\circ}$ 

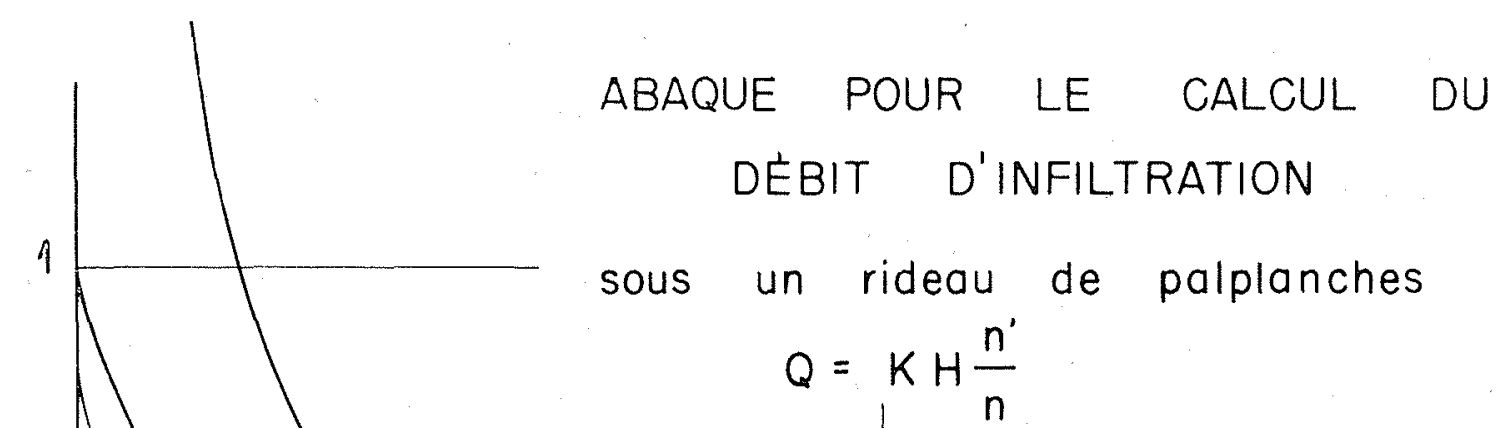

sous un rideau de palplanches

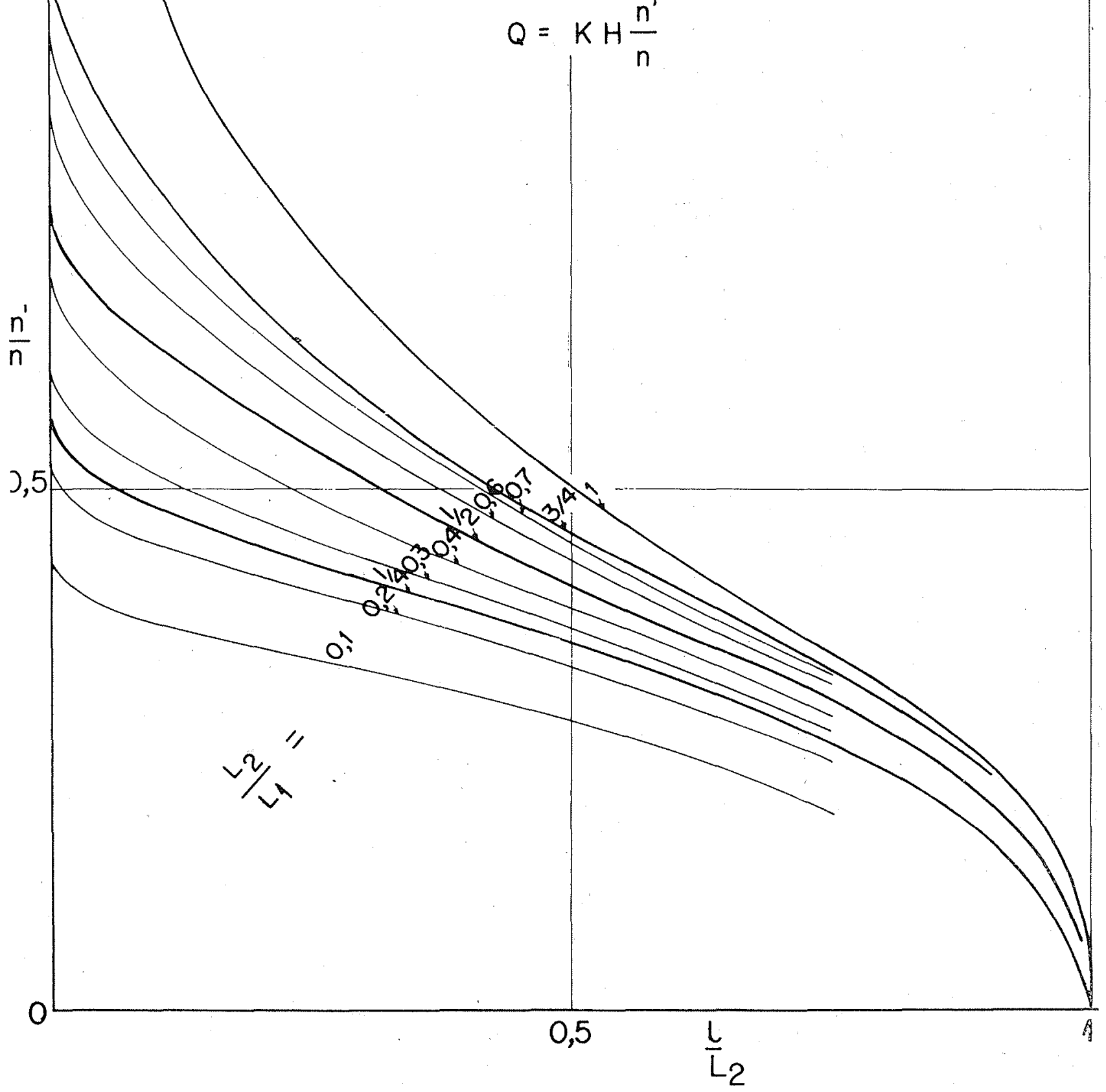

Fig. 22

aux précédentes de sorte que le nouveau, réseau obtenu est un damier D; les lettres homologues montrent comment se fait la transformation: la ligne de courant A B C D E s'étire suivant une simple droite.
La correspondance $R D$ définit une transformation conforme.

Considérons maintenant le réseau des ellipses périodiques (c'est-à-dire de l'écoulement sous un radier horizontal). Soit . $\beta \delta$ la ligne de cou- 
$B$

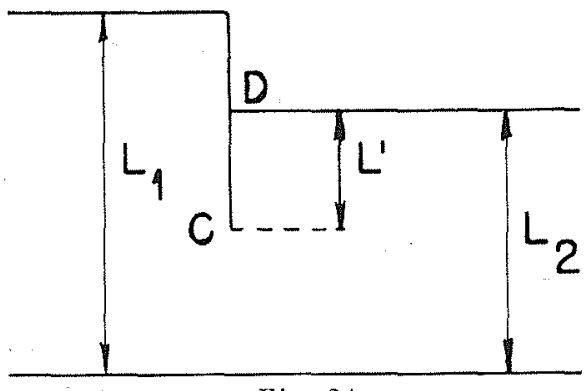

Fig. 21

rant particulière. Portons ce réseau sur le damier $D$ de manière que $\beta$ coïncide avec $\mathrm{B}$; supposons que $\beta \delta=\mathrm{B} \mathrm{D}$ et que la profondeur $L$ soit juste égale à la largeur de la bande du damier $D$.

Faisons alors subir à ce réseau des ellipses périodiques ainsi placées, la transformation $\mathrm{D} R$. Nous obtenons un cas d'écoulement qui nous intéresse particulièrement.

Le débit de fuite est encore donné par la même formule : $q=k \mathrm{H} \frac{n}{n}$.

$n$ ' est le nombre de tubes de courant et $n$ le nombre des tubes équipotentiels.

Il conviendrait, pour se rendre compte des variation's de ce débit de fuite, en fonction des différents paramètres comme nous l'avons fait précédemment, d'essayer de relier la longueur $\beta \delta$ avec les différentes longueurs $\mathrm{BC}, \mathrm{CD}$, et L. Nous n'avons pas entrepris ce travail, car il présente des difficultés de calcul. Pour chaque cas particulier, qui 'se présentera, il sera possible de construire le réseau et l'on pourra évaluer ainsi les fuites. Il est à remarquer que nous pouvons obtenir de très nombreux autres ca's d'écoulement en faisant varier la position de $\beta \delta$ par rapport à B.D.

Si nous plaçon's le segment $\beta \delta$ de manière que le segment B D lui soit intérieur, nous obtiendrons l'écoulement sous un barrage pré-

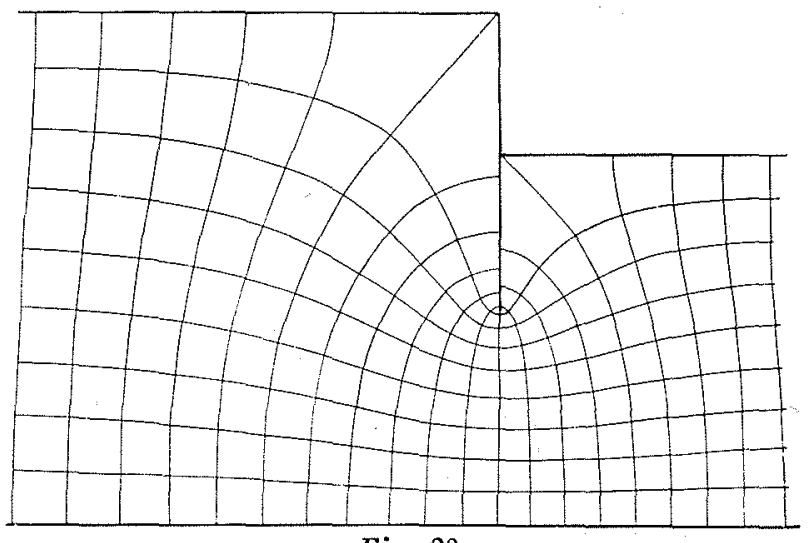

Fig. 23

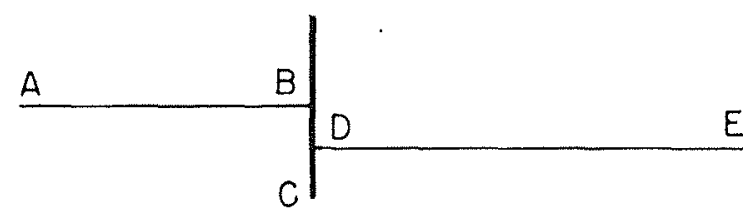

F

G
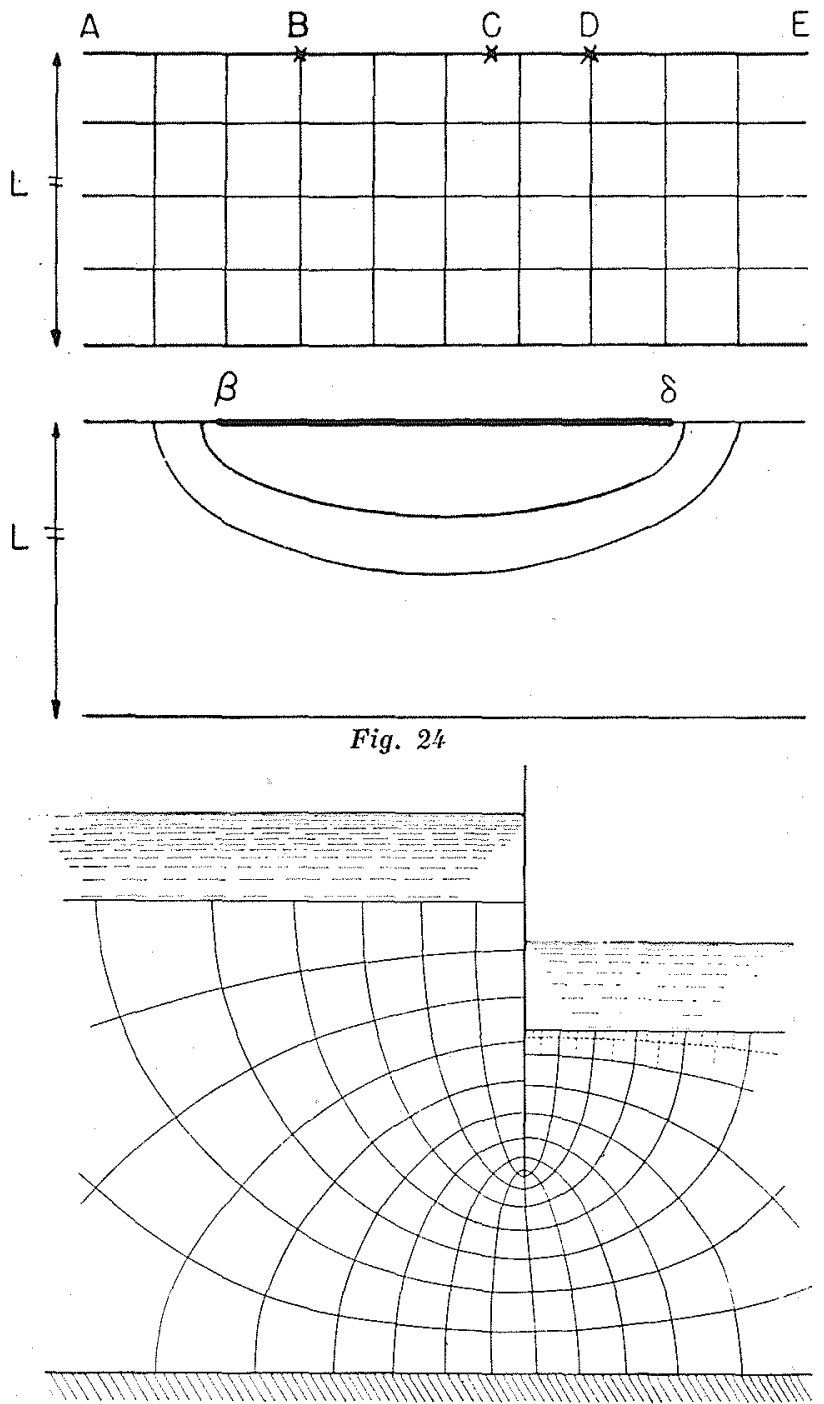

Fig. 25

cédé d'un radier horizontal, suivi d'un écran d'étanchéité, et terminé par un second radier horizontal.

Nous arrêterons ces exemples d'écoulements souterrains; le lecteur pourra, s'il le désire, appliquer cette méthode à différents autres cas. Et ce faisant, il pénétrera mieux l'intérêt du procédé ; séduit à son tour, il prendra plaisir à jouer avec ces élégantes transformations, bien mystérieuses pour le profane. 